OPEN ACCESS

Edited by:

Pietro Vajro,

University of Salerno, Italy

Reviewed by:

Dasa Longman,

University of Edinburgh,

United Kingdom

Helen M. Evans,

Starship Children's Health,

New Zealand

*Correspondence:

Yizhong Wang

wangyz@shchildren.com.cn

Ting Zhang

zhangt@shchildren.com.cn

Specialty section

This article was submitted to

Pediatric Gastroenterology,

Hepatology and Nutrition,

a section of the journal

Frontiers in Pediatrics

Received: 16 September 2020 Accepted: 23 November 2020 Published: 13 January 2021

Citation:

Jiang B, Xiao F, Li X, Xiao Y, Wang Y and Zhang T (2021) Case Report: Pediatric Recurrent Acute Liver Failure

Caused by Neuroblastoma Amplified

Sequence (NBAS) Gene Mutations.

Front. Pediatr. 8:607005

doi: 10.3389/fped.2020.607005

\section{Case Report: Pediatric Recurrent Acute Liver Failure Caused by Neuroblastoma Amplified Sequence (NBAS) Gene Mutations}

\author{
Bingxin Jiang ${ }^{1}$, Fangfei Xiao ${ }^{1}$, Xiaolu Li $^{1}$, Yongmei Xiao ${ }^{1}$, Yizhong Wang ${ }^{1,2 *}$ and \\ Ting Zhang ${ }^{1,2 *}$
}

${ }^{1}$ Department of Gastroenterology, Hepatology and Nutrition, Shanghai Children's Hospital, Shanghai Jiao Tong University, Shanghai, China, ${ }^{2}$ Institute of Pediatric Infection, Immunity and Critical Care Medicine, Shanghai Children's Hospital,

Shanghai Jiao Tong University School of Medicine, Shanghai, China

Acute liver failure (ALF) in childhood is a rapidly progressive, potentially life-threatening condition that occurs in previously healthy children of all ages. However, the etiology of $\sim 50 \%$ of cases with pediatric ALF remains unknown. We herein report a 4-year-old Chinese girl with recurrent ALF (RALF) due to a mutation in the neuroblastoma amplified sequence (NBAS) gene. The patient had suffered from multiple episodes of fever-related ALF since early childhood. She had also suffered from acute kidney injury, hypertension, mild pulmonary hypertension, pleural effusion, and hypothyroidism. A novel compound heterozygote mutation, c.3596G > A (p.C1199Y)/ex.9del (p.216-248del), in the NBAS gene was identified by whole-exome sequencing (WES). The missense mutation c.3596G > A (p. C1199Y) was inherited from her father, and ex.9del (p.216-248del) was inherited from her mother. The patient was managed with intensive treatments, such as renal replacement therapy (CRRT), intravenous antibiotics, and glucose infusion, and was discharged after full recovery. We identified a novel compound heterozygote mutation in the NBAS gene that caused fever-related RALF in a Chinese child, which further expands the mutational spectrum of NBAS.

\section{Keywords: recurrent acute liver failure, whole-exome sequencing, mutational spectrum, neuroblastoma amplified} sequence, compound heterozygote mutation

\section{INTRODUCTION}

Acute liver failure (ALF) in childhood is a rapidly progressive, potentially life-threatening condition that occurs in previously healthy children of all ages. Multiple etiologies have been identified as the causes of ALF in childhood, such as infection, inborn errors of metabolism, drug poisoning, abnormal perfusion, and autoimmune diseases. However, the etiologies of $\sim 50 \%$ of cases with pediatric ALF remain unknown (1). Recently, neuroblastoma amplified sequence (NBAS) gene mutations were identified as a novel cause of pediatric recurrent acute liver failure (RALF) by Haack et al. (2). The NBAS gene is located on chromosome 2p24.3 and has 52 exons encoding a protein containing 2,371 amino acids (NCBI GENE ID: 51594). Due to its wide expression, NBAS gene mutations can induce a wide range of clinical symptoms, ranging from isolated fever-related RALF 
to a multisystemic phenotype including short stature, skeletal defects, retinal dystrophy and optic atrophy, status epilepticus, immune dysregulation, and immunodeficiency (3). Here, we report a child with RALF caused by a novel compound heterozygous mutation in NBAS. The clinical features and genetic variants of the patient were described in this study.

\section{CASE PRESENTATION}

A 4-year-old girl with a past history of RALF was admitted to our hospital due to nausea and vomiting with a high fever $\left(39.5^{\circ} \mathrm{C}\right)$. She had no symptoms of abdominal pain and distention or diarrhea, no chills or convulsions, and no headache, dizziness, diplopia, or blurred vision. The girl was born at full-term and met normal developmental milestones. There was no family history of hereditary or metabolic liver diseases. Physical examination (PE) of the patient showed normal cognitive function without confusion, personality changes, disorientation, or consciousness disturbance. It also revealed mild jaundice, enlarged tonsils, and palpable hepatomegaly (1-2 cm below the costal margin) without splenomegaly. Nervous system examination revealed normal muscle tone and strength, a normal Babinski sign, and negativity for ankle clonus. Laboratory data showed abnormal liver function [direct bilirubin (DB) $24.50 \mu \mathrm{mol} / \mathrm{L}$ (normal range: $0-6.8$ ), total bilirubin (TB) $35.81 \mu \mathrm{mol} / \mathrm{L}$ (normal range: $3.4-$ 17.1), alanine transaminase (ALT) $7169 \mathrm{U} / \mathrm{L}$ (normal range: 5-40), aspartate transaminase (AST) $9677 \mathrm{U} / \mathrm{L}$ (normal range: 8-40), $\gamma$-glutamyl-transferase $(\gamma$-GGT) $40 \mathrm{U} / \mathrm{L}$ (normal range: 7-32 U/L), and albumin $14 \mathrm{~g} / \mathrm{L}$ (normal range: 38-54 g/L)]. The lactate dehydrogenase [>9480 U/L (normal range: 110$290 \mathrm{U} / \mathrm{L}$ )] and blood ammonia [90 $\mu \mathrm{mol} / \mathrm{L}$ (normal range: 11$51 \mu \mathrm{mol} / \mathrm{L})$ ] levels were significantly increased. Coagulopathy [prothrombin time (PT) 20.1 s (normal range: 9.8-12.1 s), international normalized ratio (INR) 1.76 (normal range: $0.82-$ 1.25), thrombin time (TT) $22.1 \mathrm{~s}$ (normal range: 14-21 s)] and renal dysfunction [serum creatinine (Cre) $524 \mu \mathrm{mol} / \mathrm{L}$ (normal range: 18-66), blood urea nitrogen (BUN) $35.6 \mathrm{mmol} / \mathrm{L}$ (normal range: 0-8.3)] were observed. Thyroid function tests were performed, with the results supporting a diagnosis of hypothyroidism [triiodothyronine (T3) $0.81 \mathrm{nmol} / \mathrm{L}$ (normal range: $1.34-3.70$ ), thyroxine (T4) $54.04 \mathrm{nmol} / \mathrm{L}$ (normal range: 64.30-158.70), free triiodothyronine (FT3) $2.84 \mathrm{pmol} / \mathrm{L}$ (normal range: 3.58-6.92), free thyroxine (FT4) $9.35 \mathrm{pmol} / \mathrm{L}$ (normal range: 9.60-14.50), and thyroid-stimulating hormone (TSH) $0.10 \mu \mathrm{IU} / \mathrm{ml}$ (normal range: $0.90-4.00$ )]. The detection of liverdamaging viruses, such as Epstein-Barr virus, cytomegalovirus, herpes simplex virus, hepatitis A virus, hepatitis B virus, and hepatitis $\mathrm{C}$ virus, was all negative. Ceruloplasmin and $\alpha 1$-antitrypsin were all normal. Tandem mass spectrometry (TMS) for metabolic disorder screening was unremarkable. Analyses of autoantibodies, including autoimmune hepatitisrelated autoantibodies, were all negative. Mitochondrial gene analysis was carried out, but no abnormalities were observed. Abdominal ultrasound showed hepatomegaly and mild ascites but no splenomegaly. Echocardiography showed mild pulmonary hypertension. The electrocardiogram (ECG) was normal. In addition, no abnormalities were observed by brain magnetic resonance imaging (MRI).

Considering that no etiological diagnosis was reached despite mitochondrial gene sequencing, the patient was subjected to whole-exome sequencing (WES) using genomic DNA extracted from peripheral blood mononuclear cells (PBMCs). Exome capture was carried out using IDTxGen Exome Research Panel (IDT, USA) and paired-end sequencing was performed on HiseqX10 (Illumina, USA). Sequencing data were analyzed to identify sequence variants [single nucleotide variant (SNV), insertion/deletion (Ins/Del)] and copy number variants (CNVs) using an in-house pipeline (Fulgent genetics). A phenotypedriven gene list was created to perform a primary variant interpretation for a more targeted analysis (4). After data analysis, variant filtering, and prioritization, a novel compound heterozygote mutation, c.3596G > A (p.C1199Y)/ex.9del (p.216$248 \mathrm{del}$ ) in NBAS was identified (Figure 1A). No other potentially pathogenic mutations were detected in genes predisposing to ALF (e.g., TRMU, OMIM: 613070; MARS OMIM:615486; LARS, OMIM: 615438; RINT1, OMIM: 618641) by WES of the patient. The mutations were verified by Sanger sequencing and quantitative PCR, respectively (Figures 1B,C). Genotyping of the unaffected parents showed the mutation c.3596G>A (p. C1199Y) in the father and the ex.9del (p.216-248del) mutation in the mother. The c.3596G > A (p. C1199Y) mutation has been previously reported in patients with NBAS deficiency (5-7), but the ex.9del (p.216-248del) mutation has not been described previously. In silico predictors predict that the variant c.3596G> A (p. C1199Y) is located in a highly conserved amino acid residue (Sec39 domain) that affects the structure/function of the NBAS protein and is defined as pathogenic (SIFT score: 0.000, http:// sift.jcvi.org; PROVEAN score: -9.563, http://provean.jcvi.org/ index.php). The ex.9 del (p.216-248del) variant located in the $\beta$ propeller leads to the deletion of exon 9 and is predicted to be deleterious by PROVEAN with a score of -123.936 (Figure 1D). The two variants were classified as likely pathogenic according to the guidelines of the American College of Medical Genetics and Genomics (ACMGG).

To further confirm the liver damage, electron microscopy of the liver biopsy was performed. As shown in Figure 2, hepatocytes were irregular in shape and size with an eccentric nucleus (Figure 2A, 3,000 $\times$, scale bar: $10 \mu \mathrm{m}$ ), capillary bile ducts were dilated, and a large number of microvilli and lysosomes were visible in hepatocytes (Figure 2B, 9,000 $x$, scale bar: $5 \mu \mathrm{m}$ ). In addition, an increased number of mitochondria with abnormal morphology and an increased density of rough endoplasmic reticulum (ER) were observed (Figure 2C, 9,000 $\times$, scale bar: $5 \mu \mathrm{m}$ ). The patient's clinical symptoms were improved soon by managing with supportive treatment, including renal replacement therapy (CRRT), thyroid hormone supplementation, intravenous antibiotics, and glucose infusion (Table 1). Finally, the patient was discharged with full recovery after 1 month of hospitalization. During the recent 9-month follow-up period, she had complete clinical and biochemical recovery and did not require any additional treatment. 
A

\section{$242,289,380$}

$242,289,400$

T GT CT GT TAT CAGT TGTAAGCAGCACCTAGAAGAAATTAGATAA T GT CT GTTAT CAGT T GTAAGCl IGCACCTAGAAGAAATTAGATAA

T GT CT GT TATCAGT T GTAAGTAGCACCTAGAAGAAAT TA GATAA T GT CT GT TAT CAGT T GTAGTAGCACCTAGAAGAAAT TAGATAA T GT CT GT TAT CAGT T GTAAG AGCACC AGAAGAAAT TAGATAA T GT CT GT TAT CAGT T GTAACAGCACCTAGAAGAAAT TAGATAA T GT CT GT TAT CAGT T GTAAGTAGACCTAGAAGAAAT TAGATAA T GT CT GT TAT CAGT T GTAAGCAGCACCTAGAAGAAAT TA GATAA T GT CT GT TAT CAGT T GTAAGT AGCACCTAGAAGAAAT TAGATAA T GT CT GT TAT CAGT T GTAAGT AGCACCTAGAAGAAAT TAGATAA T GT CT GT TAT CAGT T GTAAGCAGCACCTAGAAGAAAT TAGATAA $T G T C T$ GT TAT CAGT T GTAGCAGCACCTAGAAGAAAT TAGATAA T GT CT GT TAT CAGT T GTAAGT AGCACCTAGAAGAAAT TAGATAA $T G T C T G T T A T C A G T T$ GTAAGCAGCACCTAGAAGAAAT TAGATAA T GT CT GT TAT CAGT T G AAGCAGCACCTAGAAGAAAT TAGATAA

B

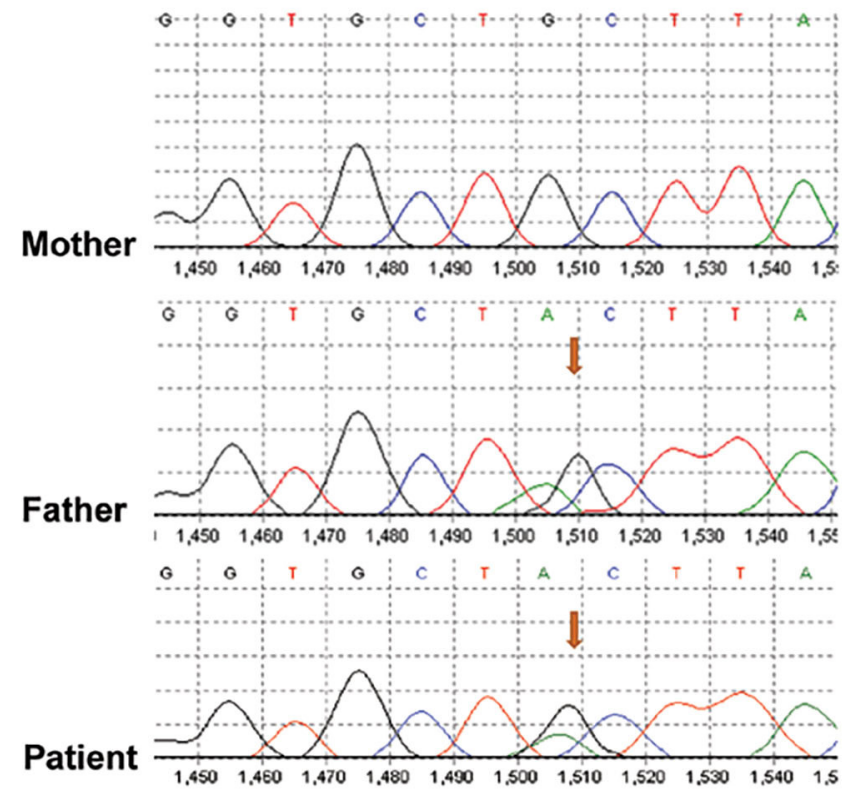

C

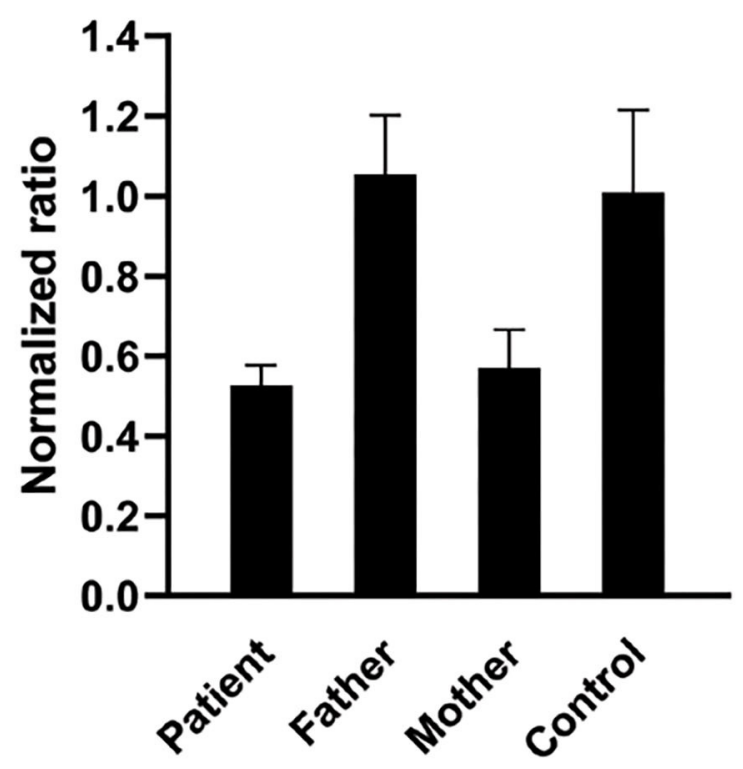

D

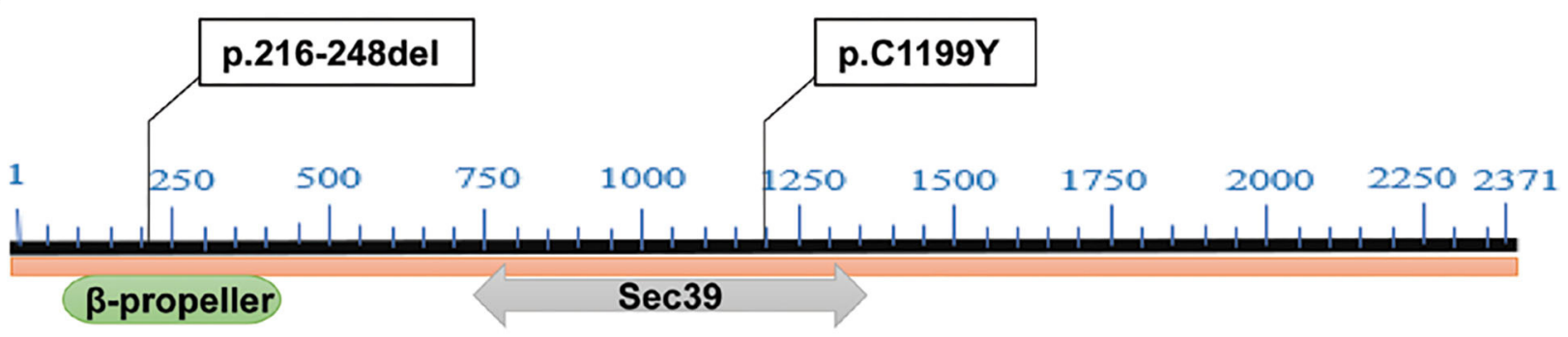

FIGURE 1 | Identification of compound heterozygous mutations in the NBAS gene in the family. (A) c.3596G>A (p.C1199Y) mutation was found by WES. (B) Sanger sequencing showed that the mutation c.3596G> A (p.C1199Y) was inherited from her father. (C) Validation of ex.9del (p.217-249del) mutation by quantitative PCR. 
FIGURE 1 | Genomic DNA was extracted from PBMCs of the patient, her parents, and healthy control. Reactions were set up using one set of primers upstream (Forward: 5'-GCTGTGTTGATTCCATGAGGA-3') and downstream (Reverse: 5'-AGTGTTGGAACAAATCAGAGCTA-3') of exon 9 in triplicate. The $\beta$-actin gene served as reference gene. The $2^{-\Delta \Delta C T}$ method was used to calculate the copy number. Sample with normalized ratio (NR) $<0.1$ denotes homozygous deletion individual, sample with NR about 0.5 denotes heterozygous deletion individual, sample with NR about 1 denotes normal individual (two copies), and sample with NR about 1.5 or more denotes copy number gain individual. (D) The location of two variants in NBAS protein: c.3596G>A (p.C1199Y) locates in C-terminal $\beta$-propeller region, and ex.9del (p.217-249del) mutation locates in the Sec39 domain.

\section{DISCUSSION}

In this report, we presented a Chinese pediatric RALF case with a novel compound heterozygote mutation, c.3596G> A (p.C1199Y)/ex.9del (p.216-248del), in the NBAS gene. In addition to the liver phenotype, she also had acute kidney injury, hypertension, mild pulmonary hypertension, pleural effusion, and hypothyroidism. Other extrahepatic manifestations, including skeletal involvement, growth failure, intellectual disability, ophthalmic abnormalities, facial dysmorphism, and cardiac abnormalities, were not observed. The c.3596G > A (p. C1199Y) mutation has been previously reported together with other mutation sites of the NBAS gene as a cause of RALF and was found to be a unique mutation among Chinese patients (5-7). A recent study $(3,7)$ reported that nine Chinese pediatric patients carried the c.3596G > A (p. C1199Y) mutation. The c.3596G >A (p. C1199Y) mutation is located in the strictly conserved Sec39 domain of NBAS and can lead to structural changes throughout the Sec39 region or the entire protein (5). The ex.9 del (p.216$248 \mathrm{del}$ ) variant is located in the $\beta$-propeller and is predicted to be deleterious in silico, which was first reported in this study. Phenotype-genotype correlation studies revealed that pediatric patients with NBAS deficiency caused by mutations in the Sec39 domain have a predominant liver phenotype (infantile liver failure syndrome type 2/ILFS2, OMIM 616483), and variants in the $\beta$-propeller usually showed liver damage combined with multiorgan/system abnormalities $(3,7)$. Our patient presented a predominant liver phenotype, which was consistent with reported NBAS deficiencies caused by mutations located in the Sec39 domain $(3,7)$. However, compared with other reported cases with mutations in the $\beta$-propeller, such as c.680690dupACTGTTTCAGC/p. Phe231ThrfsTer35, c.686dup/p. Ser230Glnfs*4, and c.680A > C/p. His227Pro (7-9), this patient had no clinical manifestation resembling SOPH syndrome. Interestingly, our patient had symptoms of hypertension, pleural effusion, and pulmonary hypertension, which further expanded the clinical spectrum of NBAS deficiency.

Currently, the exact mechanism of NBAS mutations causing RALF is not fully understood. The NBAS protein is a component of a soluble N-ethylmaleimide-sensitive factor attachment protein receptor (SNARE) complex, the syntaxin 18 complex, which is involved in Golgi-to-ER transport $(10,11)$. The NBAS protein interacts with $\mathrm{p} 31$ and ZW10-RINT-1, and it was shown that NBAS depletion reduced p31 expression and caused redistribution of Golgi recycling proteins accompanied by a defect in protein glycosylation $(2,10)$. p31 protein defects can accelerate hepatic lipogenesis and induce hepatocyte apoptosis via increasing the relative expression of stress response genes in the ER (12). The syntaxin 18 complex is thermally susceptible, as evidenced by an increased sensitivity to high temperature at the protein and functional levels and a disturbed tethering of vesicles in the skin fibroblasts of patients with NBAS mutations (2). The catabolic state and high energy consumption during fever further impair the function of the syntaxin 18 complex, resulting in ER stress (13). ER stress triggers liver cell destruction by accelerating lipogenesis and activating the unfolded protein response (14). Thus, mechanisms of fever-dependent ALF in NBAS deficiency are proposed to be associated with altered Golgi-ER retrograde transport and ER stress. In addition, NBAS plays a pivotal role in non-sense-mediated mRNA decay (NMD), a conserved posttranscriptional RNA surveillance pathway (15). NMD recognizes and degrades mRNAs bearing premature termination codons (PTCs) to avoid the accumulation of truncated proteins and their cytotoxicity (16). The NMD pathway is important for liver development, function, and regeneration (17). NBAS was found to coregulate target genes with core NMD factors and participate in a negative feedback regulatory loop in the NMD pathway (18). It was shown that NBASmediated NMD modulates genes involved in bone development and cholesterol biosynthesis, which may explain the skeletal dysplasia and Pelger-Huët anomaly in SOPH syndrome (18).

Given the rarity of RALF being caused by NBAS mutations, other genetic causes of RALF in children should be considered before diagnosis. Studies have revealed that deleterious mutations in cytosolic leucine-tRNA synthetase (LARS) and RINT1 gene mutations are common in liver failure syndrome-1 (ILFS1) (19) and liver failure syndrome-3 (ILFS3) (20), respectively. ILFS1 is characterized by the impairment of liver synthetic function, including hypoalbuminemia and severe coagulopathy, with mild or without hepatocellular injury or defects in liver detoxification during the early phase of the disease (19). The main clinical manifestations of ILFS3 are similar to ALF caused by NBAS mutations, presented with fever-related RALF, vomiting, hypoglycemia, coagulopathy, hyperammonemia, and skeletal abnormalities (20). However, there are differences in skeletal phenotypes: vertebral body abnormalities, including anterior breaking and irregularity, are common in ILFS3 (20), while NBAS deficiency has been characterized as atypical osteogenesis imperfecta with bone fragility (9). Furthermore, Wolcott-Rallison syndrome (WRS, OMIM 226980) (21) and CALFAN syndrome (OMIM 616719) $(22,23)$ should also be taken into consideration. WRS is caused by mutations in the gene encoding eukaryotic translation initiation factor $2 \alpha$ kinase 3 (EIF2AK3) and is characterized by neonatal/early-onset non-autoimmune insulin-requiring diabetes associated with skeletal dysplasia, RALF, renal dysfunction, exocrine pancreas 
A

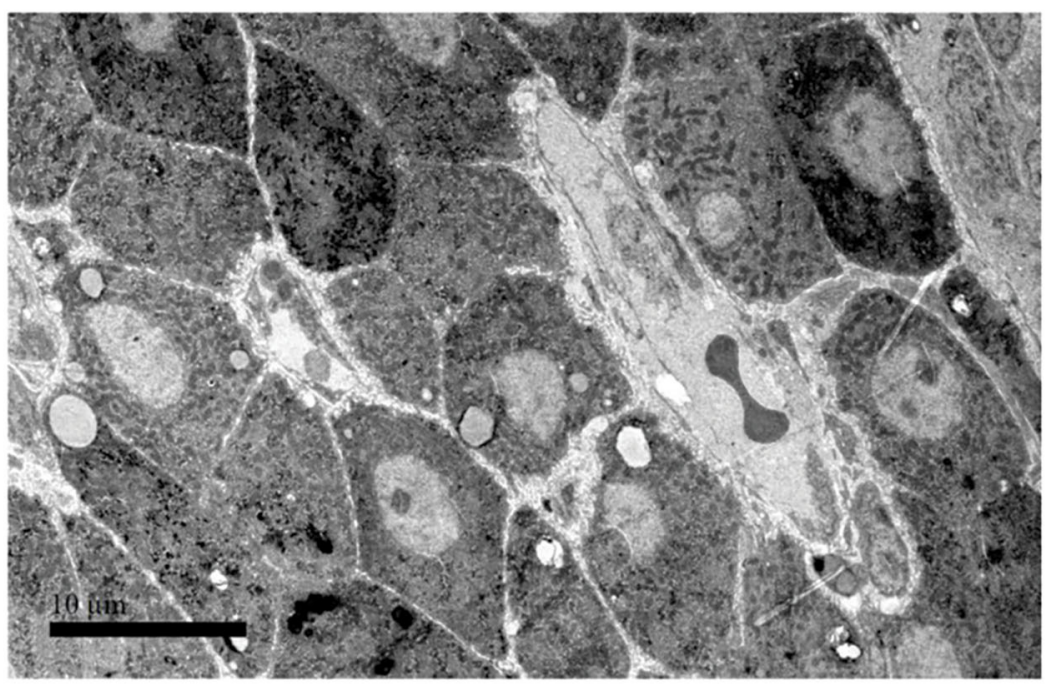

B

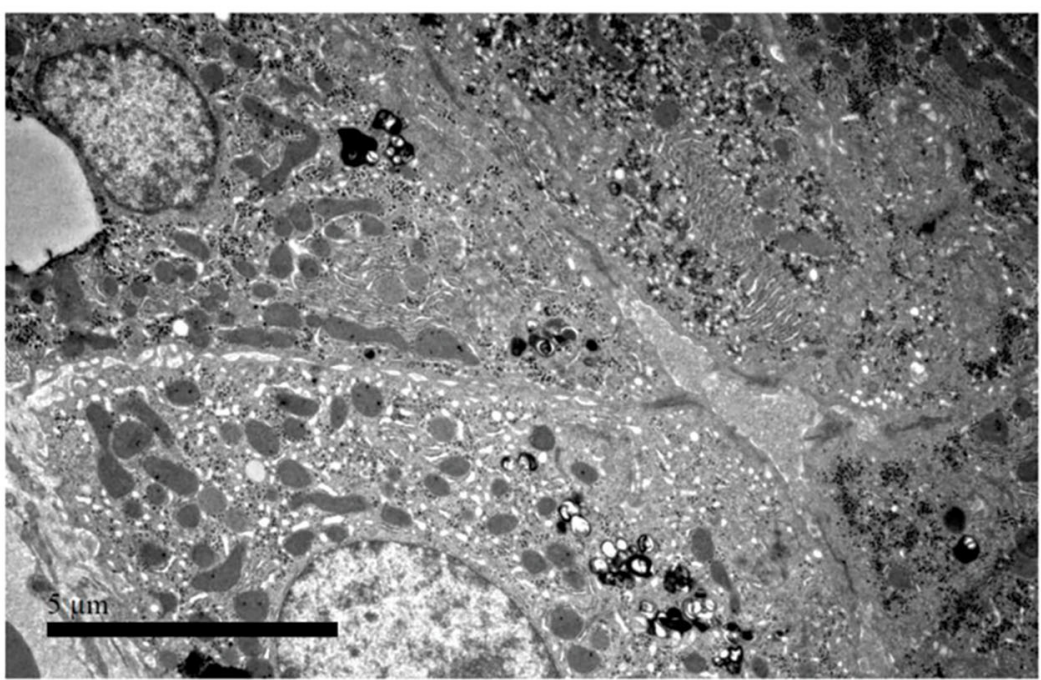

C

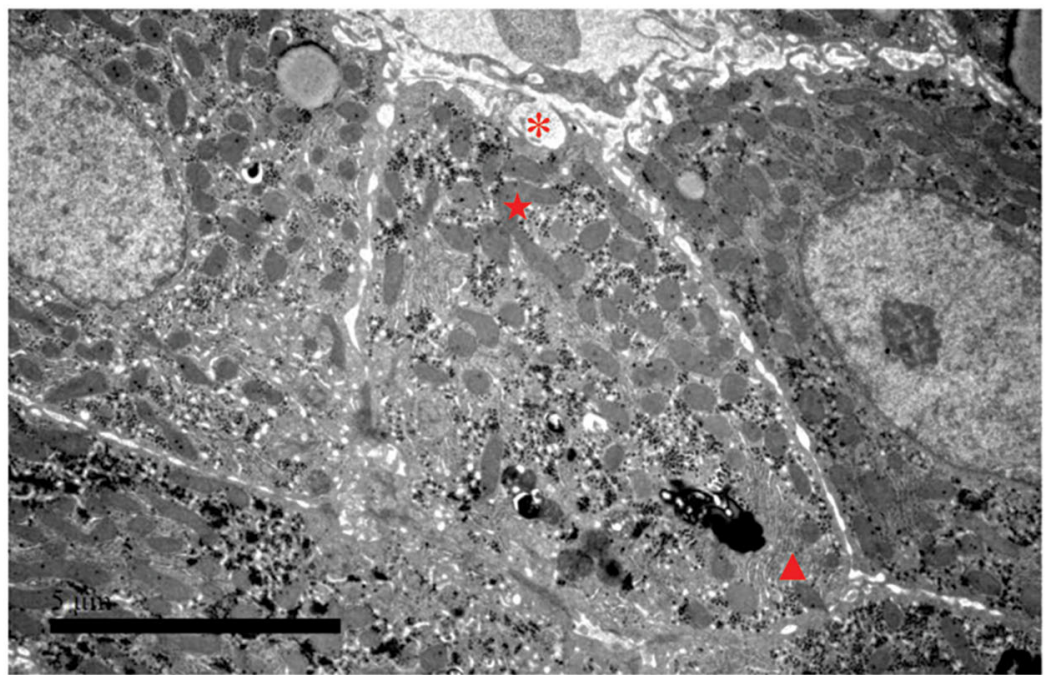

FIGURE 2 | Electron microscopy of liver biopsy. (A) Hepatocytes are irregular in shape and size, and the nucleus is eccentric (3,000 ×, scale bar: $10 \mu \mathrm{m})$. (B) Part of the capillary bile duct is dilated; a large number of microvillous and lysosomes are visible $(9,000 \times$, scale bar: $5 \mu \mathrm{m})$. (C) Hyperplasia of collagen fibers can be seen in liver cells, with cross-sections $(*)$. The picture also presents with an increased number of mitochondria with abnormal morphology $(\star)$ and an increased density of rough endoplasmic reticulum $(\mathbf{\Lambda})(9,000 \times$, scale bar: $5 \mu \mathrm{m})$. 
TABLE 1 | Laboratory findings at clinical presentation and during hospitalization.

\begin{tabular}{|c|c|c|c|c|c|c|c|c|}
\hline Parameter (normal value) & D1 & D4 & D5 & D8 & D12 & D17 & D27 & D28 \\
\hline TB (3.4-17.1 $\mu \mathrm{mol} / \mathrm{L})$ & 35.81 & 38.91 & 25.76 & 9.66 & 8.04 & 11.08 & 7.24 & 12.2 \\
\hline DB (0-6.8 $\mu \mathrm{mol} / \mathrm{L})$ & 24.5 & 35.55 & 21.7 & 7.74 & 6.24 & 3.1 & 4.59 & 5.7 \\
\hline ALT (5-40 U/L) & 7,169 & 1,189 & 772 & 381 & 176 & 91 & 29 & 25 \\
\hline AST (8-40 U/L) & 9,677 & 238 & 93 & 30 & 37 & 35 & 30 & 29 \\
\hline$\gamma$-GGT (7-32 U/L) & 40 & 97 & 86 & 113 & 100 & 120 & 65 & 61 \\
\hline Albumin (38-54 g/L) & 22 & 15 & 14 & 19 & 18 & 27 & 22 & 21 \\
\hline Blood ammonia (11-51 $\mu \mathrm{mol} / \mathrm{L})$ & 85 & 47 & 63 & 51 & 17 & 39 & & \\
\hline PT (9.8-12.1s) & 23.5 & 15 & 12.4 & 12.9 & 12.0 & 10.7 & 11.8 & 11.4 \\
\hline INR (0.82-1.25) & 2.1 & 1.32 & 1.08 & 1.13 & 1.04 & 0.93 & 1.02 & 0.98 \\
\hline Cre (18-66 $\mu \mathrm{mol} / \mathrm{L})$ & 179 & 524 & 240 & 324 & 168 & 79 & 49 & 45 \\
\hline T3 (1.34-3.70 nmol/L) & & & 0.81 & & & & & 2.3 \\
\hline T4 (64.30-158.70 nmol/L) & & & 54.4 & & & & & 109.1 \\
\hline FT3 (3.58-6.92 pmol/L) & & & 2.84 & & & & & 6.3 \\
\hline FT4 (9.60-14.50 pmol/L) & & & 9.35 & & & & & 11.5 \\
\hline TSH (0.90-4.00 ulU/mL) & & & 0.1 & & & & & 2.39 \\
\hline
\end{tabular}

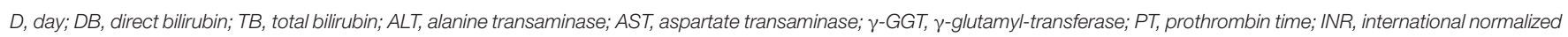
ratio; Cre, serum creatinine; T3, triiodothyronine; T4, thyroxine; FT3, free triiodothyronine; FT4, free thyroxine; TSH, thyroid-stimulating hormone.

insufficiency, intellectual deficit, hypothyroidism, neutropenia, and recurrent infections (21). Permanent early-onset diabetes is a typical manifestation of WRS (21). The clinical presentations of CALFAN syndrome included recurrent low $\gamma$-GGT cholestasis or ALF with onset in infancy and a variable neurological phenotype of later onset, which was caused by SCYL1 mutations $(22,23)$. However, the symptoms of the nervous system are less common in NBAS deficiency (9). Although those diseases involved with ALF showed similar clinical manifestations with multisystem involvement, genetic testing is valuable to help clinicians differentiate them. In addition, it needs to be distinguished from additional genetic diseases that cause ALF, such as PCK1 and PCK2 (24). In this reported case, only NBAS mutations were observed by WES, which ruled out other genetic causes of ALF.

There is no known cure treatment for patients with NBAS deficiency, and we can individualize supportive treatment and emphasize the significance of follow-up investigations. More proactive infection prevention strategies are required to avoid ALF episodes during the interval. Early and effective antipyretic therapy is necessary to prevent the later occurrence of ALF. The application of intravenous glucose and lipids can alleviate disease severity and promote recovery, as reported in the literature (9). Patients with hepatic encephalopathy (HE) or decreased serum carnitine levels can receive L-carnitine supplementation (25). ALF can be lethal in patients without treatment. Liver transplantation is the only rescue therapy for patients with endstage ALF. Thus, early diagnosis is critical for the treatment and management of pediatric ALF.

In summary, we reported that a novel compound heterozygote mutation in the NBAS gene caused fever-related RALF in a Chinese child, which further expands the mutational spectrum of NBAS. Genetic testing by NGS is valuable in the differential diagnosis of pediatric hereditary liver diseases.

\section{DATA AVAILABILITY STATEMENT}

The original contributions presented in the study are included in the article/supplementary materials, further inquiries can be directed to the corresponding author/s.

\section{ETHICS STATEMENT}

Written informed consent was obtained from the individual(s), and minor(s)' legal guardian/next of kin, for the publication of any potentially identifiable images or data included in this article.

\section{AUTHOR CONTRIBUTIONS}

TZ and YW conceived the study and edited the manuscript. BJ and YW drafted the manuscript. FX, XL, YX, and YW acquired, analyzed, and interpreted the data. All authors agreed to be accountable for all aspects of the work.

\section{FUNDING}

This work was supported by the grants from the National Natural Science Foundation of China (grant numbers 81870373 and 81900472), Shanghai Hospital Development Center New Frontier Technology Joint Research Project (grant number SHDC12017115), and Shanghai Science and Technology Committee (19140904300). The funders had no role in study design, data collection and analysis, decision to publish, or preparation of the manuscript.

\section{ACKNOWLEDGMENTS}

We are grateful to the patient's family who contributed to this study. 


\section{REFERENCES}

1. Squires RH Jr, Shneider BL, Bucuvalas J, Alonso E, Sokol RJ, Narkewicz $\mathrm{MR}$, et al. Acute liver failure in children: the first 348 patients in the pediatric acute liver failure study group. J Pediatr. (2006) 148:652-8. doi: 10.1016/j.jpeds.2005.12.051

2. Haack TB, Staufner C, Kopke MG, Straub BK, Kolker S, Thiel C, et al. Biallelic mutations in NBAS cause recurrent acute liver failure with onset in infancy. Am J Hum Genet. (2015) 97:163-9. doi: 10.1016/j.ajhg.2015.05.009

3. Staufner C, Peters B, Wagner M, Alameer S, Baric I, Broue P, et al. Defining clinical subgroups and genotype-phenotype correlations in NBASassociated disease across 110 patients. Genet Med. (2020) 22:610-21. doi: 10.1038/s41436-019-0698-4

4. Feng J, Lan X, Shen J, Song X, Tang X, Xu W, et al. A de novo MAPRE2 variant in a patient with congenital symmetric circumferential skin creases type $2 . \mathrm{Mol}$ Genet Genomic Med. (2020) 8:e1096. doi: 10.1002/mgg3.1096

5. Wang J, Pu Z, Lu Z. Targeted nextgeneration sequencing reveals two novel mutations of NBAS in a patient with infantile liver failure syndrome2. $\mathrm{Mol}$ Med Rep. (2018) 17:2245-50. doi: 10.3892/mmr.2017.8191

6. Li JQ, Qiu YL, Gong JY, Dou LM, Lu Y, Knisely AS, et al. Novel NBAS mutations and fever-related recurrent acute liver failure in Chinese children: a retrospective study. BMC Gastroenterol. (2017) 17:77. doi: 10.1186/s12876-017-0636-3

7. Li ZD, Abuduxikuer K, Zhang J, Yang Y, Qiu YL, Huang Y, et al. NBAS disease: 14 new patients, a recurrent mutation, and genotype-phenotype correlation among 24 Chinese patients. Hepatol Res. (2020) 50:1306-15. doi: 10.1111/hepr.13559

8. Regateiro FS, Belkaya S, Neves N, Ferreira S, Silvestre P, Lemos S, et al. Recurrent elevated liver transaminases acute liver failure in two siblings with novel bi-allelic mutations of NBAS. Eur J Med Genet. (2017) 60:426-32. doi: 10.1016/j.ejmg.2017.05.005

9. Staufner C, Haack TB, Kopke MG, Straub BK, Kolker S, Thiel C, et al. Recurrent acute liver failure due to NBAS deficiency: phenotypic spectrum, disease mechanisms, therapeutic concepts. J Inherit Metab Dis. (2016) 39:316. doi: 10.1007/s10545-015-9896-7

10. Aoki $\mathrm{T}$, Ichimura $\mathrm{S}$, Itoh $\mathrm{A}$, Kuramoto $\mathrm{M}$, Shinkawa $\mathrm{T}$, Isobe $\mathrm{T}$, et al. Identification of the neuroblastoma-amplified gene product as a component of the syntaxin 18 complex implicated in Golgi-toendoplasmic reticulum retrograde transport. Mol Biol Cell. (2009) 20:2639-49. doi: 10.1091/mbc.e08-11-1104

11. Jahn R, Scheller RH. SNAREs-engines for membrane fusion. Nat Rev Mol Cell Biol. (2006) 7:631-43. doi: 10.1038/nrm2002

12. Uemura T, Sato T, Aoki T, Yamamoto A, Okada T, Hirai R, et al. p31 deficiency influences endoplasmic reticulum tubular morphology and cell survival. $\mathrm{Mol}$ Cell Biol. (2009) 29:1869-81. doi: 10.1128/MCB.01089-08

13. Mallakmir S, Nagral A, Bagde A, Mirza D, Merchant R, Yewale V. Mutation in the neuroblastoma amplified sequence gene as a cause of recurrent acute liver failure, acute kidney injury, status epilepticus. J Clin Exp Hepatol. (2019) 9:753-6. doi: 10.1016/j.jceh.2019.03.008

14. Dara L, Ji C, Kaplowitz N. The contribution of endoplasmic reticulum stress to liver diseases. Hepatology. (2011) 53:1752-63. doi: 10.1002/hep.24279
15. Anastasaki C, Longman D, Capper A, Patton EE, Caceres JF. Dhx34 and Nbas function in the NMD pathway and are required for embryonic development in zebrafish. Nucleic Acids Res. (2011) 39:3686-94. doi: 10.1093/nar/gkq1319

16. Chang YF, Imam JS, Wilkinson MF. The nonsense-mediated decay RNA surveillance pathway. Annu Rev Biochem. (2007) 76:51-74. doi: 10.1146/annurev.biochem.76.050106.093909

17. Thoren LA, Norgaard GA, Weischenfeldt J, Waage J, Jakobsen JS, Damgaard I, et al. UPF2 is a critical regulator of liver development, function and regeneration. PLoS ONE. (2010) 5:e11650. doi: 10.1371/journal.pone.0011650

18. Longman D, Hug N, Keith M, Anastasaki C, Patton EE, Grimes G, et al. DHX34 and NBAS form part of an autoregulatory NMD circuit that regulates endogenous RNA targets in human cells, zebrafish and Caenorhabditis elegans. Nucleic Acids Res. (2013) 41:8319-31. doi: 10.1093/nar/gkt585

19. Peroutka C, Salas J, Britton J, Bishop J, Kratz L, Gilmore MM, et al. Severe neonatal manifestations of infantile liver failure syndrome type 1 caused by cytosolic leucine-tRNA synthetase deficiency. JIMD Rep. (2019) 45:71-6. doi: 10.1007/8904_2018_143

20. Cousin MA, Conboy E, Wang JS, Lenz D, Schwab TL, Williams M, et al. RINT1 Bi-allelic variations cause infantile-onset recurrent acute liver failure and skeletal abnormalities. Am J Hum Genet. (2019) 105:108-21. doi: 10.1016/j.ajhg.2019.05.011

21. Julier C, Nicolino M. Wolcott-Rallison syndrome. Orphanet J Rare Dis. (2010) 5:29. doi: 10.1186/1750-1172-5-29

22. Li JQ, Gong JY, Knisely AS, Zhang MH, Wang JS. Recurrent acute liver failure associated with novel SCYL1 mutation: a case report. World J Clin Cases. (2019) 7:494-499. doi: 10.12998/wjcc.v7.i4.494

23. Lenz D, McClean P, Kansu A, Bonnen PE, Ranucci G, Thiel C, et al. SCYL1 variants cause a syndrome with low gamma-glutamyl-transferase cholestasis, acute liver failure, neurodegeneration (CALFAN). Genet Med. (2018) 20:1255-65. doi: 10.1038/gim.2017.260

24. Vieira P, Cameron J, Rahikkala E, Keski-Filppula R, Zhang LH, Santra S, et al. Novel homozygous PCK1 mutation causing cytosolic phosphoenolpyruvate carboxykinase deficiency presenting as childhood hypoglycemia, an abnormal pattern of urine metabolites and liver dysfunction. Mol Genet Metab. (2017) 120:337-41. doi: 10.1016/j.ymgme.2017.02.003

25. Abbasnezhad A, Choghakhori R, Kashkooli S, Alipour M, Asbaghi O, Mohammadi R. Effect of L-carnitine on liver enzymes and biochemical factors in hepatic encephalopathy: a systematic review and meta-analysis. $J$ Gastroenterol Hepatol. (2019) 34:2062-70. doi: 10.1111/jgh.14765

Conflict of Interest: The authors declare that the research was conducted in the absence of any commercial or financial relationships that could be construed as a potential conflict of interest.

Copyright (c) 2021 Jiang, Xiao, Li, Xiao, Wang and Zhang. This is an open-access article distributed under the terms of the Creative Commons Attribution License (CC $B Y)$. The use, distribution or reproduction in other forums is permitted, provided the original author(s) and the copyright owner(s) are credited and that the original publication in this journal is cited, in accordance with accepted academic practice. No use, distribution or reproduction is permitted which does not comply with these terms. 